\title{
Dietary Nitrite: from menace to marvel
}

\begin{abstract}
Nathan S. Bryan
Department of Molecular and Human Genetics, Baylor College of Medicine, Houston, TX 77030, USA

*Corresponding author: Nathan S. Bryan, $\mathrm{PhD}$, Department of Molecular and Human Genetics, Baylor College of Medicine, One Baylor Plaza, Alkek Biomedical Building for Research, Room 830R, Houston, TX 77030, USA

Submission Date: August 2, 2016, Accepted Date: November 5, 2016, Publication Date: November 30, 2016

Citation: Nathan S. Bryan. Dietary Nitrite: from menace to marvel. Functional Foods in Health and Disease 2016; 6(11):691-701

ABSTRACT:

The health benefits of nitrite are now indisputable when administered in a clinical setting for specific diseases. Currently, most published reports identify the production of nitric oxide (NO) as the mechanism of action for nitrite. Basic science, in addition to clinical studies, demonstrate that nitrite and/or nitrate cannot restore NO homeostasis as an endothelium independent source of NO that may be a redundant system for endogenous NO production. Nitrate must first be reduced to nitrite by oral commensal bacteria; nitrite can then be further reduced to NO along the physiological oxygen gradient. But despite decades of rigorous research on sodium nitrate's safety and efficacy as a curing agent, sodium nitrite is still regarded by many as a toxic undesirable food additive. However, research within the biomedical science community has revealed enormous therapeutic benefits of nitrite which are being developed as novel therapies for conditions associated with nitric oxide insufficiency. Thus, this review will highlight the fundamental biochemistry of nitrite in human physiology and provide evidence that nitrite be considered an essential nutrient. Foods or diets enriched with nitrite can have profound positive health benefits.
\end{abstract}

Keywords: nitrite, nitrate, nitric oxide, curing, nutrition, epidemiology, cardiovascular, cancer, diet, nitrosamines, antioxidants

\section{INTRODUCTION}

Imagine if a single dietary component was proven to be able to protect one from injury from heart attack [1], reverse hypertension [2, 3], enhance insulin signaling and glucose uptake while reversing features of metabolic syndrome [4, 5], inhibit microvascular inflammation, reverse endothelial dysfunction and reduce levels of C-reactive protein [6], kill food borne pathogens [7, 8], enhance gastric mucosal blood flow [9], prevent ulcerative colitis [10] and inhibit cancer cell 
growth and proliferation $[11,12]$. These are just a few of the physiological functions of dietary nitrite which have been revealed over the past decade [13]. However, negative press over the previous 50 years has dampened the enthusiasm for these revolutionary discoveries and are consequently met with some resistance. Therefore, a change in paradigm is required. The nitrite molecule, which was once considered a potentially toxic food additive that should be avoided, is now considered to be an essential nutrient by many individuals in science. This review will highlight the health benefits of dietary nitrite, while also discussing mechanisms to prevent nitrosative chemistry and subsequent nitrosamine formation.

Nitric Oxide Biochemistry and Physiology: Before we review the underlying biochemistry of nitrite in human physiology, it is first necessary to describe the fundamental roles and production pathways for $\mathrm{NO}$ and its implications in health and disease in order to better appreciate the new found and beneficial role of nitrite. The mammalian biosynthesis of NO discovered in the 1980's for its roles in the immune [14, 15], cardiovascular [16-18], and nervous [19] systems established a new, startling paradigm in the history of cellular signaling mechanisms. Prior to this discovery, $\mathrm{NO}$ was widely considered and recognized as a toxic molecule. In the past, NO was identified as a common air pollutant, a constituent of cigarette smoke, and a toxic gas, which appears in the exhaust of motor cars and jet airplanes, causes acid rain, and destroys the ozone layer. Consequently, it was essentially inconceivable that cells would intentionally produce this toxic gas as a part of normal physiology. A similar phenomenon is now occurring regarding nitrite. Although nitrate was once considered to be a harmful and toxic food additive implicated in cancer, it is now acknowledged to be a beneficial molecule with medicinal properties that positively affect many organ systems. NO is now recognized to be one of the most important signaling molecules in the body, being involved in virtually every organ system in which it is responsible for modulating an astonishing variety of effects. The primary targets for NO are thiols [20] or iron/copper-containing proteins [16]. NO can bind to soluble gualylyl cyclase (sCG) and cause an increase in second messenger cGMP [16] and mediate a number of physiological functions. This pathway was considered to be the basis of NO based signaling, until it was discovered that $\mathrm{NO}$ elicited a number of physiological and biological effects that were not dependent upon cGMP. It is now recognized that $\mathrm{NO}$ can react directly with thiyl radicals to form nitrosothiols or other reaction products of $\mathrm{NO}$ i.e. nitrite, $\mathrm{N}_{2} \mathrm{O}_{3}$ or $\mathrm{N}_{2} \mathrm{O}_{4}$ that can post-translationally modify thiols to affect protein structure and function [21]. NO has been shown to affect and be involved with practically every organ system in the body [22]. Therefore, one can imagine how a host of diseases, conditions, and multi-systemic symptoms may be caused or affected by the body's dysregulation of NO production/signaling. Maintaining NO homeostasis is critical for optimal health and disease prevention. Nitrite may be fundamental in maintaining NO homeostasis through reduction to NO and through maintenance of nitrosothiols $[23,24]$.

The consequences of NO insufficiency are broad and profound. The continuous generation of NO is essential for the integrity of the cardiovascular system; additionally, the decreased production and/or bioavailability of NO is central to the development of many disorders [22]. Aging is considered the single largest risk factor related to cardiovascular related diseases and deaths. Cardio-protection decreases with increasing age and is attributed to a decline in NO. The 
lack of NO production can lead to hypertension, atherosclerosis, peripheral artery disease, heart failure, and thrombosis leading to heart attack and stroke, the leading cause of death for all Americans. Remarkably, all of these conditions have been shown to be positively affected by dietary nitrite interventions $[13,25,26]$.

Nitrite in Human Physiology: Although the L-arginine-NO pathway was the first to be discovered, it does not necessarily mean it is the primary pathway for the endogenous production of NO. In fact, nitrite may be central in the maintenance of NO homeostasis. The activation and metabolism of nitrite in human physiology requires hemoproteins [27], similar to the known chemistry in meat curing. This is the historical basis for the addition of sodium nitrite into readyto-eat meat and cured meat products. Research performed over the past decade realized that nitrite is physiologically recycled in blood and tissues to form $\mathrm{NO}$ and other bioactive nitrogen oxides [23, 28-30]. Nitrite is an oxidative breakdown product of NO that has been shown to serve as an acute marker of $\mathrm{NO}$ flux/formation [31]. Nitrite is in steady state equilibrium with Snitrosothiols $[23,32]$ and has been shown to activate sGC and increase cGMP levels in tissues [23], activities very similar to NO. In the early 1980's, it was demonstrated that, in addition to dietary exposure, nitrite is also generated endogenously [33]. Shortly thereafter, the entire Larginine-nitric oxide synthase (NOS)-system was discovered and found to be the major endogenous source of nitrite, since NO is rapidly oxidized to nitrite [15]. Once nitrite is produced and circulated, it is taken up by peripheral tissues and can be stored in cells. The 1electron reduction of nitrite can occur by ferrous heme proteins (or any redox active metal) through the following reaction:

$$
\mathrm{NO}_{2}{ }^{-}+\mathrm{Fe}^{(\mathrm{III})}+\mathrm{H}^{+} \quad \leftrightarrow \quad \mathrm{NO}+\mathrm{Fe}^{[\mathrm{IIII}]}+\mathrm{OH}^{-}
$$

This is the same biologically active NO which is produced by NOS, with nitrite rather than L-arginine as the precursor. However, nitrite reduction along the physiological oxygen gradient is a relatively inefficient process [24]. It is now clear that nitrite is recycled in vivo and again forms bioactive nitrogen oxides, including NO [35-40]. Thus, instead of simply wasting the products of $\mathrm{NO}$ oxidation, mammals store and actively recycle it. Human nitrite reduction to NO was first described in the stomach, where salivary nitrite forms NO non-enzymatically via acidcatalyzed reduction $[29,30]$. Shortly after this observation, Zweier described NOS-independent nitrite reduction in the ischemic and acidic heart [41]. In the last 15 years, it has become evident that blood and tissue nitrite is reduced under physiological conditions to form $\mathrm{NO}$ and nitrosothiols and modulate blood flow [42, 43].

Much of the recent focus on nitrite physiology is due to its ability to be reduced to NO during ischemic or hypoxic events [44-46]. Nitrite reduction in mammalian tissues has been linked to the mitochondrial electron transport system [47, 48], protonation [45], deoxyhemoglobin [43], and xanthine oxidase [49, 50]. Nitrite can also transiently form nitrosothiols (RSNOs) under both normoxic and hypoxic conditions (Figure 1) [44]. For example, a recent study by Bryan et al demonstrates that steady state concentrations of tissue nitrite and nitroso species are affected by changes in dietary nitrite intake [23]. However, nitrite reduction to NO is very effectively inhibited by oxygen [23, 24], thereby leading to the 
fundamental question as to how effective nitrite reduction is along the physiological oxygen gradient. Nonetheless, research clearly demonstrates the enriching dietary intake of nitrite results in significantly less injury from heart attack [1].
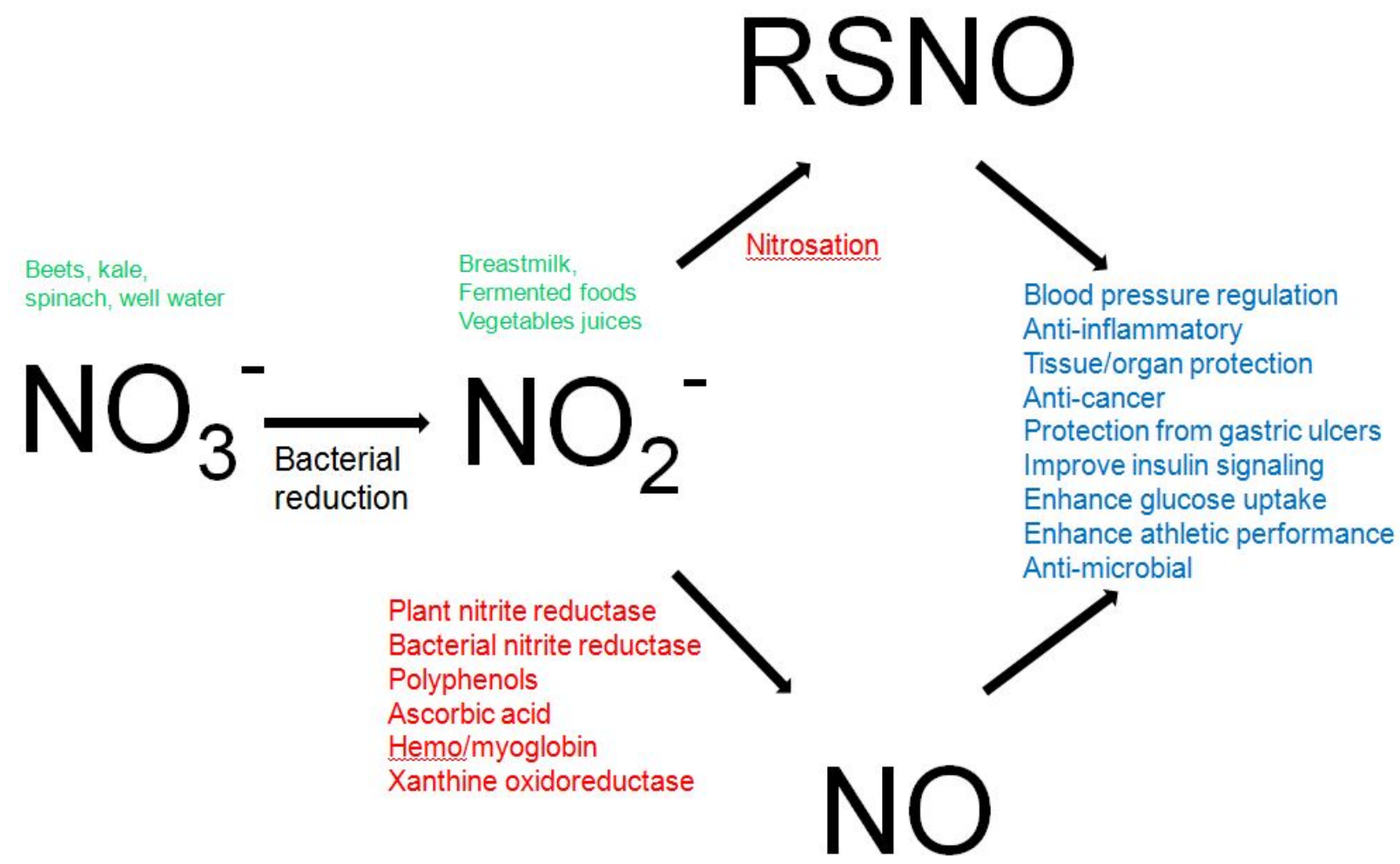

Figure 1: Nitrite is formed from nitrate through bacterial metabolism and can be found naturally in certain foods. Nitrite can form S-nitrosothiols (RSNO) and can be reduced to nitric oxide (NO). Both NO and RSNO have been shown to normalize blood pressure and exert a number of health promoting and disease preventing activities.

Published studies have also demonstrated how nitrite therapy given intravenously prior to reperfusion can protect against hepatic and myocardial ischemia/reperfusion (I/R) injury [51]. Additionally, experiments in primates have revealed a beneficial effect of long-term application of nitrite on cerebral vasospasm [52]. Moreover, inhalation of nebulized nitrite selectively dilates the pulmonary circulation under hypoxic conditions in vivo in sheep [53]. Topical application of nitrite improves skin infections and ulcerations [54]. As in meat products, the oxidation of lipids is prevented by nitrite in humans, primarily by NO binding to heme proteins, lowering the amount of free iron available to catalyze initiation and propagation steps of lipid oxidation. Studies by Carr and Frie [55] have demonstrated that myeloperoxidase mediated low density lipid modification is inhibited by nitrite. Furthermore, in the stomach nitrite-derived NO appears to play an important role in host defense [56] and the regulation of gastric mucosal integrity [57]. Nitrite has also been shown to completely prevent microvascular inflammation and endothelial dysfunction resulting from a high fat, high cholesterol diet [6] and reduce C-reactive protein. Chronic sodium nitrite therapy augments ischemia-induced angiogenesis and arteriogenesis, thereby demonstrating that sodium nitrite therapy is a recently discovered therapeutic treatment 
for peripheral artery disease and critical limb ischemia [58]. Most recently, a dietary supplement formulated with dietary nitrite and natural product chemistry to reduce nitrite to NO has been shown to modify cardiovascular risk in patients over the age of 40 , reduce blood pressure and reduce markers of inflammation [59]. All of these studies, alongside the observation that nitrite can act as a marker of NOS activity [31] opened a new avenue for the diagnostic and therapeutic application of nitrite, especially in cardiovascular diseases, using nitrite as marker as well as an active agent. Accordingly, a report by Kleinbongard et al. [60] demonstrates that plasma nitrite levels progressively decrease with increasing cardiovascular risk. Since a substantial portion of steady state nitrite concentrations in blood and tissue are derived from dietary sources [23], modulation of nitrite intake may provide a first line of defense for conditions associated with NO insufficiency [46].

Toxicity of Nitrite and Nitrate: Historically there are three measures of safety and toxicity regarding nitrite. Those are methemoglobinemia, hypotension, and formation of N-nitrosamines. Acute toxicity is defined by methemoglobinemia. The fatal dose of nitrite due to methemoglobinemia is in the range of $22-23 \mathrm{mg} / \mathrm{kg}$ body weight (from USFDA Generally Recognized as Safe Food Ingredient: Nitrates and Nitrites (Including Nitrosamines) 1972 by Battele-Columbus Laboratories and Department of Commence, Springfield VA). This dose is approximately 150 times higher than doses which have been used therapeutically in humans. Lower doses of either nitrite or nitrate have caused acute methemoglobinemia, particularly in infants where a high nitrite or nitrate intake has been associated with "blue baby syndrome" caused by methemoglobinemia [61-63]. Although infants are more susceptible to methemoglobinemia, due to the reduced activity of methemoglobin reductase [64] in adults weights of $70-80 \mathrm{~kg}$, the amount of nitrite needed for acute toxicity is $1650 \mathrm{mg}$. Therefore, only extremely high concentrations of nitrite can cause methemoglobinemia.

Hypotension or low blood pressure is another potential safety concern with nitrite exposure. However, nitrite is a very poor vasodilator especially at doses that are used to recapitulate NO based signaling [65-67]. Interestingly, nitrite appears to only lower blood pressure in subjects with an elevation in blood pressure [68]. As a result, this creates safe and effective levels for therapy with the mitigation of side effects or unwanted symptoms.

N-Nitrosamines are potent carcinogens and can be formed from nitrite in the presence of low molecular weight amines. Thus, the public health concerns are actually related to the formation of carcinogenic N-Nitrosamines rather than to the nitrite itself. About 30 different animal species are responsive to the carcinogenic effects of approximately 300 different Nnitrosamines [69]. Nitrite is not the only source of N-nitrosamines; exposure can occur in occupational settings, through one's diet, cosmetics, tobacco products, and agricultural chemicals. N-nitrosamine formation in cured meat products occurs when amines react with nitrogen oxides such as nitrite in an acid environment (i.e. the stomach) or when heated to very high temperatures (i.e. bacon frying).

During an infection or inflammation, products of nitric oxide including nitrite are generated that react with the amines by nitrosation reactions to form $\mathrm{N}$-nitrosamines, thereby causing human exposure to N-nitrosamines and possibly linking chronic inflammation to certain cancers. The nitrosation of amines in the body can also be catalyzed by certain bacteria with nitrite to form the N-nitrosamines. The carcinogenicity of N-nitrosamines is due to a very critical 
cytochrome P450- mediated metabolic activation step. Although there is evidence to support a plausible biological mechanism for formation of $\mathrm{N}$-nitrosamines, there are also numerous effective inhibitors of $\mathrm{N}$-nitrosation reactions in biological systems [70]. It was discovered that ascorbic acid (vitamin C) very potently inhibits N-nitrosamine formation [71]. Another antioxidant, alpha-tocopherol (vitamin E), has also been shown to inhibit N-nitrosamine formation [72]. Ascorbic acid, erythorbic acid and alpha-tocopherol inhibit N-nitrosamine formation due to their oxidation-reduction properties. For example, when ascorbic acid is oxidized to dehydroascorbic acid, nitrous anhydride, a potent nitrosating agent formed from sodium nitrite, is reduced to NO, which is not a nitrosating agent. Stoichiometrically, one molecule of ascorbic acid can reduce two molecules of acidified nitrite to NO [73, 74]. However, in the presence of dissolved oxygen, NO can be oxidized back to nitrite/nitrous acid. This recycling means that more than half the molar equivalent of ascorbic acid compared to nitrite is required to prevent formation of $N$-nitroso compounds. In other words, for every mole of nitrite, one mole of ascorbate is needed to yield one mole of nitric oxide, plus another 0.5 mole ascorbate to prevent the back reaction. The ratio of ascorbic acid to nitrite is recognized as a major determinant of the generation of N-nitroso compounds within the acidic lumen of the stomach [73]. Contemporary meat-curing methods use ascorbic acid or erythorbate to prevent Nnitrosation reactions and to facilitate the curing process. Furthermore, any vegetable based nitrite or nitrate source typically has sufficient polyphenols or vitamin $\mathrm{C}$ to naturally prevent nitrosative chemistry. This is the inherent protective nature of nitrite from natural sources.

\section{CONCLUSION}

The emerging health benefits of nitrite represent a profound change in the established paradigm which has existed for the past 50 years. Until the present, scientists have operated under the paradigm of the L-arginine-NO pathway by NOS enzymes as the only pathway to produce NO. There are a number of recycling pathways to regenerate NO from dietary nitrite. The emergence of a redundant pathway for maintenance of $\mathrm{NO}$ homeostasis by dietary nitrite provides a new mode of intervention and a new paradigm for restoring NO homeostasis. Nitrite therapy or supplementation may restore NO homeostasis from endothelial dysfunction and provide benefits for a number of diseases characterized by NO insufficiency $[13,75]$. If so, this will provide the basis for new preventive or therapeutic strategies and new dietary guidelines for optimal health. There are currently a number of clinical trials using sodium nitrite as a therapeutic agent (please view the following link for more information: www.clinicaltrials.gov). From a public health perspective, we may be able to make better recommendations on diet and dramatically affect the incidence and severity of cardiovascular disease, in addition to subsequent clinical events. Replenishing nitrite through dietary means may then act as a protective measure to compensate for insufficient NOS activity under conditions of hypoxia or in a number of conditions characterized by NO insufficiency. Studies using a patented formulation (US patents 8,303,995, $8,298,589,8,435,5708 \quad 8,962,038 \quad 9,119,823 \& 9,241,999$ ) in the form of an orally disintegrating tablet found that it could modify cardiovascular risk factors in patients over the age of 40, significantly reduce triglycerides, and reduce blood pressure [59]. This same lozenge was used in a pediatric patient with argininosuccinic aciduria and significantly reduced his blood pressure when prescription medications were ineffective [76]. A more recent clinical trial using the nitrite lozenge reveals that a single lozenge can significantly reduce blood pressure, dilate 
blood vessels, improve endothelial function and arterial compliance within hypertensive patients [68]. Furthermore, in a study of pre-hypertensive patients (BP $>120 / 80<139 / 89)$, administration of one lozenge twice daily led to a significant reduction in blood pressure $(12 \mathrm{mmHg}$ systolic and $6 \mathrm{mmHg}$ diastolic) after 30 days [77]. Most recently, this nitrite lozenge was also demonstrated to reduce carotid plague by $11 \%$ over six months [78]. Although it appears this dietary supplement is safe and effective in short term studies, long term studies are needed. There are a host of diseases which are associated with decreased NO availability as measured by nitrite. The enormous benefit of exogenous dietary nitrite is becoming more evident in a number of disease models, in both animals and humans. The active agent of some medicinal foods may indeed be nitrite, as nitrite is present in many green leafy vegetables, breast milk, beet root, and many other foods that have shown to be protective from many diseases. The historical classification of nitrite as a "cure" may now have new meaning.

\section{REFERENCES:}

1. Bryan, N.S., et al., Dietary nitrite supplementation protects against myocardial ischemia-reperfusion injury. Proc Natl Acad Sci U S A, 2007. 104(48): p. 19144-9.

2. Tsuchiya, K., et al., Dietary nitrite ameliorates renal injury in L-NAME-induced hypertensive rats. Nitric Oxide, 2010. 22(2): p. 98-103.

3. Kapil, V., et al., Inorganic nitrate supplementation lowers blood pressure in humans: role for nitrite-derived NO. Hypertension, 2010. 56(2): p. 274-81.

4. Jiang, H., et al., Dietary nitrite improves insulin signaling through GLUT4 translocation. Free Radic Biol Med, 2014. 67: p. 51-7.

5. Carlstrom, M., et al., Dietary inorganic nitrate reverses features of metabolic syndrome in endothelial nitric oxide synthase-deficient mice. Proc Natl Acad Sci U S A, 2010.

6. Stokes, K.Y., et al., Dietary nitrite prevents hypercholesterolemic microvascular inflammation and reverses endothelial dysfunction. Am J Physiol Heart Circ Physiol, 2009. 296(5): p. H1281-8.

7. Bjorne, H., E. Weitzberg, and J.O. Lundberg, Intragastric generation of antimicrobial nitrogen oxides from saliva--physiological and therapeutic considerations. Free Radic Biol Med, 2006. 41(9): p. 1404-12.

8. Dykhuizen, R.S., et al., Antimicrobial effect of acidified nitrite on gut pathogens: importance of dietary nitrate in host defense. Antimicrob Agents Chemother, 1996. 40(6): p. 1422-5.

9. Bjorne, H.H., et al., Nitrite in saliva increases gastric mucosal blood flow and mucus thickness. J Clin Invest, 2004. 113(1): p. 106-14.

10. Ohtake, K., et al., Oral nitrite ameliorates dextran sulfate sodium-induced acute experimental colitis in mice. Nitric Oxide, 2010. 23(1): p. 65-73.

11. Morcos, E., et al., Inhibition of cancer cell replication by inorganic nitrite. Nutr Cancer. 62(4): p. 501-4.

12. Jiang, H., et al., Concentration- and stage-specific effects of nitrite on colon cancer cell lines. Nitric Oxide, 2012. 26(4): p. 267-73.

13. Bryan, N.S. and J. Loscalzo, eds. Nitrite and Nitrate in Human Health and Disease. Nutrition and Health, ed. A. Bendich. 2011, Humana Press: New York. 
14. Stuehr, D.J. and M.A. Marletta, Mammalian nitrate biosynthesis: mouse macrophages produce nitrite and nitrate in response to Escherichia coli lipopolysaccharide. Proc Natl Acad Sci U S A, 1985. 82(22): p. 7738-42.

15. Hibbs, J.B., Jr., R.R. Taintor, and Z. Vavrin, Macrophage cytotoxicity: role for Larginine deiminase and imino nitrogen oxidation to nitrite. Science, 1987. 235(4787): p. 473-6.

16. Arnold, W.P., et al., Nitric oxide activates guanylate cyclase and increases guanosine 3':5'-cyclic monophosphate levels in various tissue preparations. Proc Natl Acad Sci U S A, 1977. 74(8): p. 3203-7.

17. Ignarro, L.J., et al., Endothelium-derived relaxing factor produced and released from artery and vein is nitric oxide. Proc. Natl. Acad Sci. USA, 1987. 84: p. 9265-9269.

18. Furchgott, R.F. and J.V. Zawadzki, The obligatory role of endothelial cells in the relaxation of arterial smooth muscle by acetycholine. Nature, 1980. 288(5789): p. 373376.

19. Garthwaite, J., S.L. Charles, and R. Chess-Williams, Endothelium-derived relaxing factor release on activation of NMDA receptors suggests role as intercellular messenger in the brain. Nature, 1988. 336(6197): p. 385-8.

20. Stamler, J.S., et al., S-nitrosylation of proteins with nitric oxide: synthesis and characterization of biologically active compounds. Proc Natl Acad Sci USA, 1992. 89: p. 444-448.

21. Foster, M.W., T.J. McMahon, and J.S. Stamler, S-nitrosylation in health and disease. Trends Mol Med, 2003. 9(4): p. 160-8.

22. Moncada, S., R.M.J. Palmer, and A. Higgs, Nitric oxide: physiology, pathophysiology and pharmacology. Pharmacol Rev, 1991. 43(2): p. 109-142.

23. Bryan, N.S., et al., Nitrite is a signaling molecule and regulator of gene expression in mammalian tissues. Nat Chem Biol, 2005. 1(5): p. 290-7.

24. Feelisch, M., et al., Tissue Processing of Nitrite in Hypoxia: An Intricate Interplay of Nitric Oxide-Generating and -Scavenging Systems. J Biol Chem, 2008. 283(49): p. 33927-34.

25. Lundberg, J.O., et al., Nitrate and nitrite in biology, nutrition and therapeutics. Nat Chem Biol, 2009. 5(12): p. 865-9.

26. Lundberg, J.O., E. Weitzberg, and M.T. Gladwin, The nitrate-nitrite-nitric oxide pathway in physiology and therapeutics. Nat Rev Drug Discov, 2008. 7(8): p. 156-167.

27. Gladwin, M.T., J.H. Crawford, and R.P. Patel, The biochemistry of nitric oxide, nitrite, and hemoglobin: role in blood flow regulation. Free Radic Biol Med, 2004. 36(6): p. 707-17.

28. Zweier, J.L., et al., Enzyme-independent formation of nitric oxide in biological tissues. Nat Med, 1995. 1(8): p. 804-809.

29. Lundberg, J.O., et al., Intragastric nitric oxide production in humans: measurements in expelled air. Gut, 1994. 35(11): p. 1543-6.

30. Benjamin, N., et al., Stomach NO synthesis. Nature, 1994. 368(6471): p. 502.

31. Kleinbongard, P., et al., Plasma nitrite reflects constitutive nitric oxide synthase activity in mammals. Free Radical Biology \& Medicine, 2003. 35(7): p. 790-796. 
32. Angelo, M., D.J. Singel, and J.S. Stamler, An S-nitrosothiol (SNO) synthase function of hemoglobin that utilizes nitrite as a substrate. Proc Natl Acad Sci U S A, 2006. 103(22): p. 8366-71.

33. Green, L.C., S.R. Tannenbaum, and P. Goldman, Nitrate synthesis in the germfree and conventional rat. Science, 1981. 212(4490): p. 56-8.

34. Cosby, K., et al., Nitrite reduction to nitric oxide by deoxyhemoglobin vasodilates the human circulation. Nature Medicine, 2003. 9: p. 1498-1505.

35. Lundberg, J.O., E. Weitzberg, and M.T. Gladwin, The nitrate-nitrite-nitric oxide pathway in physiology and therapeutics. Nat Rev Drug Discov, 2008. 7(2): p. 156-67.

36. Weitzberg, E. and J.O. Lundberg, Nonenzymatic nitric oxide production in humans. Nitric Oxide, 1998. 2(1): p. 1-7.

37. Lundberg, J.O., et al., Nitrate, bacteria and human health. Nat Rev Microbiol, 2004. 2(7): p. 593-602.

38. Lundberg, J.O. and E. Weitzberg, NO generation from nitrite and its role in vascular control. Arterioscler Thromb Vasc Biol, 2005. 25(5): p. 915-22.

39. Gladwin, M.T., et al., The emerging biology of the nitrite anion. Nat Chem Biol, 2005. 1(6): p. 308-14.

40. Zweier, J.L., A. Samouilov, and P. Kuppusamy, Non-enzymatic nitric oxide synthesis in biological systems. Biochim Biophys Acta, 1999. 1411(2-3): p. 250-62.

41. Zweier, J.L., et al., Enzyme-independent formation of nitric oxide in biological tissues. Nature Med, 1995. 1: p. 804-809.

42. Gladwin, M.T., et al., Role of circulating nitrite and S-nitrosohemoglobin in the regulation of regional blood flow in humans. Proc Natl Acad Sci U S A, 2000. 97(21): p. $11482-11487$.

43. Cosby, K., et al., Nitrite reduction to nitric oxide by deoxyhemoglobin vasodilates the human circulation. Nat Med, 2003. 9(12): p. 1498-505.

44. Bryan, N.S., et al., Cellular Targets and Mechanisms of Nitros(yl)ation: An Insight into Their Nature and Kinetics in vivo. Proc. Natl. Acad Sci. USA, 2004. 101(12): p. 4308-4313.

45. Zweier, J.L., et al., Enzyme-independent formation of nitric oxide in biological tissues. Nat Med, 1995. 1(8): p. 804-9.

46. Bryan, N.S., Nitrite in nitric oxide biology: Cause or consequence? A systems-based review. Free Radic Biol Med, 2006. 41(5): p. 691-701.

47. Walters, C.L., R.J. Casselden, and A.M. Taylor, Nitrite metabolism by skeletal muscle mitochondria in relation to haem pigments. Biochim Biophys Acta, 1967. 143(2): p. 310-8.

48. Kozlov, A.V., K. Staniek, and H. Nohl, Nitrite reductase activity is a novel function of mammalian mitochondria. FEBS Lett, 1999. 454(1-2): p. 127-30.

49. Li, H., et al., Characterization of the effects of oxygen on xanthine oxidase-mediated nitric oxide formation. J Biol Chem, 2004. 279(17): p. 16939-46.

50. Webb, A., et al., Reduction of nitrite to nitric oxide during ischemia protects against myocardial ischemia-reperfusion damage. Proc Natl Acad Sci U S A, 2004. 101(37): p. 13683-8.

51. Duranski, M.R., et al., Cytoprotective effects of nitrite during in vivo ischemiareperfusion of the heart and liver. J Clin Invest, 2005. 115(5): p. 1232-40. 
52. Pluta, R.M., et al., Nitrite infusions to prevent delayed cerebral vasospasm in a primate model of subarachnoid hemorrhage. Jama, 2005. 293(12): p. 1477-84.

53. Hunter, C.J., et al., Inhaled nebulized nitrite is a hypoxia-sensitive NO-dependent selective pulmonary vasodilator. Nat Med, 2004. 10: p. 1122-1127.

54. Hardwick, J.B., et al., A novel method for the delivery of nitric oxide therapy to the skin of human subjects using a semi-permeable membrane. Clin Sci (Lond), 2001. 100(4): p. 395-400.

55. Carr, A.C. and B. Frei, The nitric oxide congener nitrite inhibits myeloperoxidase/H2O2/ Cl- -mediated modification of low density lipoprotein. J Biol Chem, 2001. 276(3): p. 1822-8.

56. Duncan, C., et al., Chemical generation of nitric oxide in the mouth from the enterosalivary circulation of dietary nitrate. Nat Med, 1995. 1(6): p. 546-51.

57. Bjorne, H.H., et al., Nitrite in saliva increases gastric mucosal blood flow and mucus thickness. J Clin Invest, 2004. 113(1): p. 106-114.

58. Kumar, D., et al., Chronic sodium nitrite therapy augments ischemia-induced angiogenesis and arteriogenesis. Proc Natl Acad Sci U S A, 2008. 105(21): p. 7540-5.

59. Zand, J., et al., All-natural nitrite and nitrate containing dietary supplement promotes nitric oxide production and reduces triglycerides in humans. Nutr Res, 2011. 31(4): p. 262-9.

60. Kleinbongard, P., et al., Plasma nitrite concentrations reflect the degree of endothelial dysfunction in humans. Free Radic Biol Med, 2006. 40(2): p. 295-302.

61. Comly, H.H., Cyanosis in infants caused by nitrates in well water. JAMA, 1945. 129: p. 112-116.

62. Donohoe, W.E., Cyanosis in infants with drinking water as a cause. Paediatrics, 1949. 3: p. 308-311.

63. Lecks, H.I., Methemoglobinemia in infancy. Am J Dis Child, 1950. 79: p. 117-123.

64. Nilsson, A., et al., Inverse relationship between age-dependent erythrocyte activity of methaemoglobin reductase and prilocaine-induced methaemoglobinaemia during infancy. Br J Anaesth, 1990. 64(1): p. 72-6.

65. Furchgott, R.F. and S. Bhadrakom, Reactions of strips of rabbit aorta to epinephrine, isopropylarterenol, sodium nitrite and other drugs. J Pharmacol Exp Ther, 1953. 108(2): p. 129-43.

66. Bryan, N.S. and J.L. Ivy, Inorganic nitrite and nitrate: evidence to support consideration as dietary nutrients. Nutr Res, 2015. 35(8): p. 643-54.

67. Lauer, T., et al., Plasma nitrite rather than nitrate reflects regional endothelial nitric oxide synthase activity but lacks intrinsic vasodilator action. Proc. Natl. Acad Sci. USA, 2001. 98(22): p. 12814-12819.

68. Houston, M. and L. Hays, Acute effects of an oral nitric oxide supplement on blood pressure, endothelial function, and vascular compliance in hypertensive patients. J Clin Hypertens (Greenwich), 2014. 16(7): p. 524-9.

69. Hecht, S.S., Approaches to cancer prevention based on an understanding of $N$ nitrosamine carcinogenesis. Proc Soc Exp Biol Med, 1997. 216(2): p. 181-91.

70. Bartsch, H., et al., Inhibition of nitrosation. Basic Life Sci, 1993. 61: p. 27-44.

71. Mirvish, S.S., Blocking the formation of N-nitroso compounds with ascorbic acid in vitro and in vivo. Ann N Y Acad Sci, 1975. 258: p. 175-80. 
72. Mirvish, S.S., Inhibition by vitamins $C$ and $E$ of in vivo nitrosation and vitamin $C$ occurrence in the stomach. Eur J Cancer Prev, 1996. 5 Suppl 1: p. 131-6.

73. Archer, M.C., et al., Reaction of nitrite with ascorbate and its relation to nitrosamine formation. J Natl Cancer Inst, 1975. 54(5): p. 1203-5.

74. Licht, W.R., S.R. Tannenbaum, and W.M. Deen, Use of ascorbic acid to inhibit nitrosation: kinetic and mass transfer considerations for an in vitro system. Carcinogenesis, 1988. 9(3): p. 365-72.

75. Bryan, N.S., ed. Food, Nutrition and the Nitric Oxide Pathway: Biochemistry and Bioactivity. 2009, DesTech Publishing: Lancaster, PA. 238.

76. Nagamani, S.C., et al., Nitric-oxide supplementation for treatment of long-term complications in argininosuccinic aciduria. Am J Hum Genet, 2012. 90(5): p. 836-46.

77. Biswas, O.S., V.R. Gonzalez, and E.R. Schwarz, Effects of an Oral Nitric Oxide Supplement on Functional Capacity and Blood Pressure in Adults With Prehypertension. J Cardiovasc Pharmacol Ther, 2014.

78. Lee, E., Effects of Nitric Oxide on Carotid Intima Media Thickness: A Pilot Study. Alternative Therapies in Health and Medicine, 2016. 22(S2): p. 32-34. 